\title{
SORTING MOLECULES
}

$\mathrm{A}^{\mathrm{T}}$ $T$ the British Association meeting in Bristol Section B (Chemistry) arranged a session, held on September 5, at which the subject of "Sorting Molecules" was discussed. An introduction was given by the chairman, Prof. W. Baker (Bristol), who referred to the importance of sorting molecules by physical processes, and to the earliest methods of crystallization, distillation and sublimation. The first two of these are still by far the most used, and reference was made by Prof. Baker to fractionating columns, distillation in vacua, molecular stills and falling-film evaporators. The potentialities of codistillation with non-8queous liquids have been little explored. Other methods of sorting molecules are selective surface adsorption, gaseous diffusion, sedimentation by means of the ultracentrifuge, electrophoresis, and the method of distribution between two incompletely miscible solvents which has reached a high state of perfection in the Craig counter-current distribution machine.

During recent years the methods of chromato. graphy have become commonplace in almost every chemical laboratory. In its various forms, there are elution and displacement chromatography on columns of adsorbents, or on liquid phases suspended on solids or paper. The extension of adsorption and displacement chromatography to vapours is the technique of gas chromatography, and its delicacy and rapidity mark it as perhaps the most important recent advance in analytical procedures. A further method for sorting molecules, depending on molecular size and shape only, is by means of inclusion compounds, in which a 'guest' molecule is included in cavities or tunnels in the crystal of a 'host' molecule. These holes show a remarkable selectivity in accom. modating some molecules and rejecting others, and may even be capable of sorting right- and left-handed forms of the same molecule. A related method is the use of a semi-permeable membrane in dialysis; but a much more selective method is to use as a sieve a crystal lattice containing holes of molecular dimensions which can be penetrated by molecules of suitable size and shape.

In the first paper, which was on "Gas Chromatography", Dr. F. H. Pollard (Bristol) said that some indication of the importance of this new method has been the speed with which many industrial laboratories have adopted and developed this technique. The principle used in gas chromatography consists in causing each substance in a mixture to migrate at a different rate through a column of powdered material. This is achieved by one of two methods : adsorption chromatography, that is, differential adsorption on a solid such as charcoal or alumina; or gas-liquid partition chromatography, that is, differential partition of the vapours between a liquid phase held on an inert powder and a gas such as nitrogen or hydrogen flowing over the solid. For example, a mixture of hydrocarbons is admitted to the top of a column of material, and a stream of nitrogen is then passed downwards through the column, carrying the hydrocarbons with it. If the conditions of operation have been arranged correctly, the components of the mixture will leave the column one by one, and their detection as separated components depends mainly upon instruments which record differences in the physical properties of the gas stream as it emerges from the column. The two physical properties which have been extensively used for this purpose are thermal conductivity and density. Automatic recording can be readily employed, and the emergence of the different components of a mixture is indicated by a series of peaks automatically recorded on paper. From such records the individual components of a mixture can be identified and quantitatively estimated. The time required for the complete analysis can be as little as twenty minutes. For mixtures such as those of fatty acids, automatic titration equipment has been used, and for others simple volume measurements have been employed. At the meeting, demonstrations of separations using the thermal conductivity cell as a method of detection were given on two different commercial machines, arranged by Dr. B. W. Bradford (Imperial Chemical Industries, Ltd., Billingham), and a demonstration of the gas density balance was given by Mr. D.H. Desty (Research Department, British Petroleum Co.) through the courtesy of Dr. S. F. Birch.

Adsorption chromatography has been used for the separation of hydrocarbons and a wide range of organic compounds, using either frontal analysis, displacement analysis, or elution analysis on either carbon or silica gel. Janak has devised a very simple form of apparatus in which a 1-3 ml. sample is passed through a column in a stream of carbon dioxide and the volumes of the separated components measured in an azotometer over potassium hydroxide solution. This method has been applied to rare gases as well as to many organic compounds.

The development of gas-liquid partition chromato. graphy in 1952 by James and Martin marked a great advance in technique which has led to a large number of applications. Since the first separation of milligram amounts of fatty acids on a column of kieselguhr at $137^{\circ} \mathrm{C}$. carrying as static phase a mixture of liquid silicone and stearic acid, there have been extensive developments for the separation of aliphatic amines, pyridine homologues, methyl esters of higher fatty acids, halogenated hydrocarbons, organic nitrates and nitrites, and boron hydrides. Highertemperature columns are being developed, and have already been used for $\mathrm{C}_{12}-\mathrm{C}_{22}$ fatty-acid esters. Methods have been described for identifying unknown organic compounds by comparing retention volumes on two or more columns containing different static phases, which allow of different extents of interaction between the substance and the static phase. This technique of analysis has been applied to problems in chemical kinetics; for example, thermal decomposition of ethyl nitrate; studies in air pollution; identifying polymers by analysing pyrolysis products ; the preparation of radiochemically labelled fine chemicals ; and the large-scale separation of highly fluorinated organic compounds.

In the second paper at the meeting, Mr. H. M. Powell (Oxford) considered the problem of "Sorting Molecules by Shape and Size". Several methods, in particular those of X-ray crystallography, reveal that different substances are made up of molecules or other groupings of atoms. Their shapes and sizes 
may be accurately measured, and there is the possibility of separating molecules by sorting, after observation of their shapes and sizes.

Some substances have very large molecules; but many molecules contain only a few atoms, and have no dimension exceeding a ten-millionth of an inch. It is not, therefore, a simple matter to sort molecules, and if they are to be separated by their shapes it must be by something of the same order of size as the molecules themselves-that is, it must be done by other molecules or atoms.

Whenever a crystal grows from a mixture of substances as in a solution, it is automatically separating some particles from others; the crystal grows by adding particles of the same kind. It may not com. pletely reject all others because, within limits, lattices may tolerate other atoms or molecules which are not too dissimilar.

Some substances are able to form crystalline in. elusion compounds. The atoms of one component build up a structure which has in it cavities large enough to contain molecules of a second substance. Structurally there are two main types: either the cavities are effectively closed ; or they are open, long channels. These structures sort molecules by size and shape because they can contain only those molecules which are of suitable size. Closed cavities may contain molecules up to certain critioal sizes and will exclude longer ones, whereas channel structures may include both long and short molecules, but will not take in the shortest ones because the van der Waals interaction between enclosed and enclosing materials is too weak.

Urea adducts form a honeycomb-like structure with long molecules in the channels, and have been used to separate straight-chain from branched-chain aliphatic molecules which are too broad. Separations with up to 95 per cent efficiency have been reported. The method has been applied to compounds with up to thirty carbon atoms which cannot be distilled; for example, wax esters may be separated from glycerides. $\quad C i s$ and trans isomers of some compounds have also been separated. The nickel complex, $\mathrm{Ni}(\mathrm{CN})_{2} \cdot \mathrm{NH}_{3} \cdot \mathrm{C}_{6} \mathrm{H}_{6}$, has been used to obtain benzene of $99.992 \pm 0.004$ per cent purity by a very simple operation.

Some inclusion structures do not give good separa. tions. Thus, 'Dianin's compound' (4- $p$-hydroxyphenyl-2:2:4-trimethylchroman) includes a great variety of molecules; its cavities are very large and therefore not selective. Tri-o-thymotide, which also gives many inclusion compounds, forms enclosing structures held together by van der Waals forces only. In this case the cavities are adaptable to some extent to the included molecule. Although a particular tri-o-thymotide structure may occur only with included molecules the dimensions of which lie within certain limits, this does not prevent formation of adducts with other molecules with a different form of tri-o-thymotide enclosure. In such circumstances separations through addition compounds may be possible but are not likely to be efficient. There is, however, a form of separation for which tri-a. thymotide is very suitable. It normally crystallizes as a racemate, but in many of its adducts it is spontaneously resolved into a conglomerate of dextro or loevo crystals. The cavities in these crystals are, like the molecules, enantiomorphous, and may include preferentially either dextro or loevo molecules. Since tri-o-thymotide racemizes rapidly in solution, it is possible to remove only the one enantiomorphous form of crystal from a solution and to recover from it the included substance. In this way racemic secondary butyl bromide has been resolved, and the efficiency of the process approaches 100 per cent. Optical resolutions may also be carried out with urea adducts ; the process depends on the left- or righthanded spiral arrangement of the enclosing urea molecules, but seems to require many repetitions. The cyclodextrins have also been used for optical separation.

The enclosing components described have been found largely by accident. Once such a structure is known, it is possible to foresee which molecules it is likely to accept or reject and thus to employ inclusion compounds for separation purposes. It is not so easy to predict which molecules will form enclosing structures. It is desirable to have cavities which can be modified to fit a particular enclosable molecule, and the observation of large cavities in some inclusion compounds suggests how this might be done. If a molecular arrangement analogous to that of $\beta$-quinol but with much larger cavities could be built up, it might be possible to vary the cage-forming component by the addition of a substituent without drastic change in the main structure.

A major problem in industrial and academic science is how to separate the multitudes of chemical species from one another, by neat, simple and effective methods, and new principles for doing this are constantly being sought. A novel method consists in using crystal lattices as molecular sieves, and this was discussed in the third, and last, paper, given by Prof. R. M. Barrer (Imperial College, London), his paper being entitled the "Separation of Molecular Mixtures using Crystal Sieves". A crystal with an open lattice is employed quite literally to sift molecules. Those molecules of the wrong shape or size are held back, while others of appropriate form pass easily into the crystal, which then occludes them copiously. The separations achieved are fully quantitative in a great many cases, by the single, clear-cut operation of exposing the crystal powder to the molecular mixture, or filtering the molecular mixture through a bed of the crystals. An account was given of some of the work done in Prof. Barrer's laboratory in introducing the molecular sieve method, and in characterizing, operating, modifying and synthesizing crystal sieves of various kinds.

Naturally occurring crystal sieves were discovered among the crystalline zeolites. In these species, which are all aluminosilicates, $\mathrm{SiO}_{4}$ or $\mathrm{AlO}_{4}$ tetrahedra are built into more complex structural units, often polyhedra. These polyhedra may have considerable cavities at their centres (chabazite), or it may be the stacking of the polyhedra which creates new and larger cavities (faujasite; natrolite, thomsonite, scolecite). Windows open into the cavities and provide ready means of entry or exit for sorbed molecules and means of transit from one cavity to another. These windows also determine the molecular sieve properties of the crystal as a whole. By sharing of oxygen atoms, the polyhedra are built into threedimensional structures which often display remarkable rigidity and permanence, while being permeated by cavities interconnected by windows. The volume of the cavities may be more than 50 per cent of the volume of the crystal. Four major categories of crystal sieve were then characterized by Prof. Barrer, using certain molecules of known dimensions as yardsticks. A general order of openness of the crystal lattices in a few cases is : faujasite $>$ calcium-rich 
chabazite $>$ sodium-rich mordenite $>$ calcium-rich mordenite or calcium-rich levynite $>$ analcite.

Faujasite, when freed of its crystal water by heat and evacuation, freely occludes iso-paraffins and simple aromatics and naphthenes ; chabazite will not occlude iso-paraffins, aromatics and naphthenes, but does occlude $n$-paraffins, and molecules of smaller cross-section; sodium mordenite will not occlude $n$-paraffins, from $\mathrm{C}_{3}$ upwards, but sorbs $\mathrm{C}_{1}$ and $\mathrm{C}_{2}$ paraffins, argon atoms, and nitrogen, oxygen and similar small molecules; while calcium-mordenite or levynite does not sorb even $\mathrm{C}_{1}$ and $\mathrm{C}_{2}$ paraffins, and yet still occludes nitrogen, oxygen and similar small molecules. Typical quantitative separations, using the appropriate crystal sieve, have been made, inter alia, of aromatics, naphthenes or iso-paraffins from $n$-paraffins ; of mono-substituted methanes and ethanes with amine, hydroxyl, chloride, bromide or cyanide as substituents from similar mono-substituted $n$-paraffins having the substituent group at a secondary carbon atom; of mono-substituted methanes from similar mono-substituted ethanes; or of simple inorganic species (water, ammonia) from organic species. Mixtures which are azeotropic, or in which the constituents have virtually the same boiling point, can readily be separated if appropriate size and shape differences arise between their molecules (for example, water-ethyl alcohol; or $n-\mathrm{C}_{7} \mathrm{H}_{16}-$ iso- $\mathrm{C}_{8} \mathrm{H}_{18}$ ).

The crystal sieves have been modified, within limits set by a given aluminosilicate framework, by ion exchanges of the type : $2 \mathrm{Na} \rightleftharpoons \mathrm{Ca}$ (change of valence), $\mathrm{Na} \rightleftharpoons \mathrm{K}$ (change in radius of cation); and by more complex isomorphous replacements such as $\mathrm{NaAl} \rightleftharpoons \mathrm{Si}$. The sieve characteristics can also be changed by leaving small amounts of polar molecules (for example, water, ammonia, methylamine) in the crystal lattice. In these ways a great diversification of sieve types can be effected, and an almost continuous gradation of such sieves now appears possible. Finally, a substantial number of zeolites has been synthesized by hydrothermal growth from gels, representative of all main classes of sieve. Many of these zeolites have no natural counterparts, and raise interesting problems in crystal chemistry and crystal growth.

\title{
INFLUENCE OF SPACE FLIGHT ON ENGINEERING AND SCIENCE*
}

\author{
By MILTON W. ROSEN \\ Naval Research Laboratory, Washington, D.C.
}

$\mathrm{W}$ ITHIN the past few years many scientists have predicted seriously and confidently that human beings from the Earth would, in the foreseeable future, travel to the Moon and the nearer planets. The ranks of those who would dispute this prospect are diminishing rapidly. Although much of the progress is still guarded by military necessity, space flight is emerging as an activity in its own right-one that can command the efforts of many engineers and scientists.

In the United States the exploration of the upper atmosphere, the frontier to space, is being conducted vigorously. Pilots of rocket aircraft have experienced conditions approximating to those in free space, if only for a few minutes. The effect of space flight upon the human organism is being investigated-the U.S. Air Force maintains a Department of Space Medicine. There is an international organization devoted to the promotion of space travel, and there are space flight societies in twenty-three countries.

The ancients, except for a few rare individuals with greater insight, conceived of the world as an enclosure ; they stood upon the Earth at the bottom and gazed upward at a blue ceiling upon which a multitude of lights, a few great and many small, seemed to move under the influence of an unseen hand. The atmosphere filled this enclosure, and it was believed that if man had wings he could fly to the ceiling and determine the source of the lights. If a few philosophers guessed more nearly at the truth, certainly the average man had no better conception of the universe than the fanciful picture just described. There could be no valid idea of space flight until Copernieus, Kepler and Galileo placed the Earth in

- Substance of a paper read before Section G (Engineering) at the Bristol meeting of the British Assoctation on September 1 . its true relation to the universe and at the same time gave dimensions to space.

When at last the Moon and the planets were found to be material bodies not unlike our Earth, it was possible to ponder whether the immense separating distances could be traversed by man or any manmade device. The situation was made even more discouraging when, in 1686, Newton ${ }^{1}$ defined the nature and the magnitude of gravitational attraction. If previously there had been some fanciful hope of visiting celestial bodies, now surely it appeared that man was destined to remain for ever a prisoner of his own planet. In view of the great distances, it seemed unlikely that the atmosphere could extend through interplanetary space, and any suspicion that it might was laid to rest when Torricelli's barometer was carried to a mountain-top and taken aloft by the early balloonists.

Although Newton brought man face-to-face with one formidable aspect of the problem, namely, gravity, he also provided, in his three laws of motion, the key that would unlock the door to space. The fundamental equation of rocket action in free space and hence of space flight:

$$
V_{b}=C \cdot \ln \frac{M_{0}}{M_{b}}
$$

where $V_{b}$ is velocity of rocket at the end of burning, $C$ is velocity of exhaust jet, $M_{0}$ is initial mass of rocket, and $M_{b}$ is mass of rocket at the end of burning, is derived by integrating Newton's third law of motion. Nevertheless, more than two centuries were to pass before anyone performed the integration or realized that the simplest embodiment of Newton's third law, a rocket, is the only machine capable of propelling itself in a vacuum. 